Sección Médica / Medical

Artículos de investigación / Research paper

\title{
Detección de malatión en larvas de dípteros de importancia forense en el noreste de México
}

\author{
Detection of malathion in dipterous larvae of forensic importance in northeast Mexico
}

\author{
ISRAEL ESTRADA-CAMACHO ${ }^{1}$; JOSÉ LUIS HERNÁNDEZ-MENDOZA²; \\ JORGE TORRES-ORTEGA'; JESÚS DI CARLO QUIROZ-VELÁZQUEZ ${ }^{4}$; \\ KARLA VILLARREAL-SOTELO ${ }^{5}$
}

\begin{abstract}
${ }^{1} \mathrm{Ph}$. D. Fiscalía General de la República. Carretera Reynosa-Monterrey km 211+500, Cd. Reynosa, Tamaulipas, México, israelforense@gmail.com, https:// orcid.org/0000-0001-6192-9348. ${ }^{2} \mathrm{Ph}$. D. IPN-Instituto Politécnico Nacional. Centro de Biotecnología Genómica, Boulevard del Maestro S/N esq. Elías Piña, Col. Narciso Mendoza, Cd. Reynosa, Tamaulipas, México, jhernandezm@ipn.mx, https://orcid.org/0000-0002-1233-0133. ${ }^{3}$ Ph. D. IPN-Instituto Politécnico Nacional. Centro de Biotecnología Genómica, Boulevard del Maestro S/N esq. Elías Piña, Col. Narciso Mendoza, Cd. Reynosa, Tamaulipas, México, jatorreso31@gmail.com, https://orcid.org/0000-0001-8022-9638. ${ }^{4} \mathrm{Ph}$. D. IPN-Instituto Politécnico Nacional. Centro de Biotecnología Genómica, Boulevard del Maestro S/N esq. Elías Piña, Col. Narciso Mendoza, Cd. Reynosa, Tamaulipas, México, jquiroz@ipn.mx, https://orcid.org/0000-0002-6021-0427. ${ }^{5}$ Ph. D. Universidad Autónoma de Tamaulipas, Reynosa-Aztlán, calle 16 y Lago de Chapala, Col. Aztlán, Cd. Reynosa, Tamaulipas, México, kvillar@uat.edu.mx, https://orcid.org/0000-0002-8668-3257.
\end{abstract}

Autor para correspondencia: Israel Estrada-Camacho. Ph. D. Fiscalía General de la República. Carretera Reynosa-Monterrey km $211+500$, Cd. Reynosa, Tamaulipas, México, israelforense@gmail.com, https://orcid.org/00000001-6192-9348.

Citación sugerida / Suggested citation: ESTRADA-CAMACHO, I.; HERNÁNDEZMENDOZA，J. L.; TORRES-ORTEGA，J.; QUIROZ-VELÁZQUEZ, J.C.; VILLARREALSOTELO, K. 2020. Detección de malatión en larvas de dípteros de importancia forense en el noreste de México. Revista Colombiana de Entomología 46 (1): e10166. https://doi. org/10.25100/socolen.v46i1.10166

Recibido: 29-mar-2018

Aceptado: 12-sep-2019

Publicado: 29-jul-2020

Revista Colombiana de Entomología

ISSN (Impreso): 0120-0488

ISSN (En línea): 2665-4385

https://revistacolombianaentomologia.univalle.edu.co/

Open access

Publicadores / Publishers:

Sociedad Colombiana de Entomología

SOCOLEN (Bogotá, D. C., Colombia)

http://www.socolen.org.co

Universidad del Valle (Cali, Colombia)

http://www.univalle.edu.co/

(C) 2020 Sociedad Colombiana de Entomología - SOCOLEN y Universidad del Valle - Univalle
Resumen: La intoxicación aguda con plaguicidas es un problema de salud pública mundial. En los países en desarrollo los plaguicidas causan hasta un millón de casos de intoxicación y hasta 20.000 muertes anuales. En México, se ha reportado que debido a las intoxicaciones por xenobióticos, entre ellos los plaguicidas, fallecen 1.400 personas anualmente. En el presente trabajo se optimizó un método para detectar y cuantificar malatión en larvas de mosca (Diptera) de tercer estadio, que eclosionaron sobre carne de cerdo Sus scrofa domesticus tratada con la dosis letal de malatión para humanos $(60 \mathrm{~g} / 60 \mathrm{~kg})$. Se trabajó con una biomasa de $1,5 \mathrm{~g}$ a la cual se le agregó $1,2 \mathrm{~mL}$ de fase, se agitó por espacio de 5 min y se centrifugó por $10 \mathrm{~min}$ a $10.000 \mathrm{rpm}$. El sobrenadante se analizó por HPLC, usando una fase de metanol-agua (70/30). El tiempo de retención del malatión fue de 4,1 min. Se encontró que el límite de detección fue de $0,301 \mathrm{ppm}$ de malatión; la técnica y el procedimiento utilizados servirán como herramienta indirecta para identificar la presencia de este tóxico de una manera relativamente sencilla y su uso en casos judiciales.

Palabras clave: Diptera, entomotoxicología, insecticida, intoxicación, organofosforado.

Abstract: Acute pesticide poisoning is a global public health problem. In developing countries pesticides cause up to one million cases of poisoning and up to 20,000 deaths annually. In Mexico, it has been reported that due to intoxications by xenobiotics, among them pesticides, 1,400 people die annually. In the present work a method was optimized to detect and quantify malathion in third instar fly larvae (Diptera) that hatched in pork meat, Sus scrofa domesticus, treated with the lethal dose in humans of malathion $(60 \mathrm{~g} / 60 \mathrm{~kg})$. A biomass of $1.5 \mathrm{~g}$ which was added $1.2 \mathrm{~mL}$ of phase, stirred by space $5 \mathrm{~min}$ and centrifuged for $10 \mathrm{~min}$ at 10.000 $\mathrm{rpm}$. The supernatant was analyzed by HPLC, using a methanol-water phase $(70 / 30)$. The retention time of malathion was $4.1 \mathrm{~min}$. The detection limit was calculated at $0.301 \mathrm{ppm}$. The technique and procedure used will serve as an indirect tool to identify the presence of the toxin in a relatively simple way in court cases.

Keywords: Diptera, entomotoxicology, insecticide, intoxication, organophosphorus.

\section{Introducción}

La entomología forense en general es aceptada como una herramienta esencial cuando se investigan casos de homicidios, (Magni et al. 2014). La entomología médico legal o médico criminal son términos adecuados para hacer referencia de los insectos y otros artrópodos de un individuo en estado de descomposición o cadáver y cuyo resultado del análisis puede ser usado como una evidencia o indicio en una investigación criminalística (Quiroz y Rodríguez 2015).

La muerte de una persona involucra cambios y transformaciones fisicoquímicas que producen con ello una comunidad de organismos necrófagos, necrófilos, omnívoros y accidentales asociados a los procesos de descomposición del cadáver (Sarmiento y Padilla 2014) siendo los artrópodos los más frecuentes, entre los que destacan los insectos (Smith 1986). Estos son atraídos rápidamente por el cadáver debido a los pronunciados olores emanados durante la descomposición de la materia 
orgánica, concurriendo principalmente del orden Diptera y Coleoptera (Oliva 2001) y en menor incidencia Hymenoptera y Lepidoptera (Arnaldos et al. 2005) seguidos de arácnidos y nematodos (Campobasso et al. 2001).

La ciencia forense reconoce y acepta cinco fases o estados de descomposición, estos corresponden al estado fresco o cromático, hinchado o enfisematoso, descomposición activa o colicuativa, descomposición avanzada y restos secos o esqueletización (Magaña 2001; Wolff et al. 2001). Por lo anterior, diversos estudios demuestran que el orden Diptera representado por las familias Calliphoridae, Sarcophagidae y Muscidae, es predominante en los procesos de sucesión en las fases de descomposición tempranas, sobre todo porque aprovechan los cadáveres como sustratos para la ovoposición y fuente de alimento de las larvas (Goff et al. 2004).

Con los métodos tradicionales de toxicología, los productos de envenenamiento se recuperan mediante muestras de órganos como hígado, pulmón, sangre y orina; sin embargo, en estados avanzados de descomposición esto no es posible (Introna et al. 2001). De ahí que los insectos puedan ser una alternativa en la detección de sustancias en un cuerpo en descomposición (Gosselin et al. 2011). La entomotoxicología, mediante el uso de artrópodos presentes en el cuerpo sin vida puede ayudar a identificar cualitativa y cuantitativamente de forma indirecta sustancias como drogas, metales pesados, fármacos y plaguicidas (Liu et al. 2009).

Los plaguicidas organofosforados son uno de los químicos más comúnmente utilizados para el control de insectos debido a su alta eficacia y amplio espectro de efectividad. El uso inapropiado e ilegal de ellos incrementa el riesgo de exposición humana (Wang et al. 2012). Con relación a lo anterior, es importante señalar que la intoxicación aguda con plaguicidas es un problema de salud pública mundial ya que, aproximadamente, se han documentado 300.000 muertes por año ocasionadas por estas sustancias. La mayoría de estos decesos ocurre por la exposición a compuestos organofosforados, organoclorados y al fósforo de aluminio (Goel y Aggarwal 2007).

En México se ha reportado que, debido a las intoxicaciones por xenobióticos, anualmente fallecen 1.400 personas (72\% de los casos son accidentales y $28 \%$ corresponden a suicidios) (Pimentel et al. 2005). En este contexto, conviene señalar que recientes estudios realizados en el estado de Aguascalientes, los suicidios han aumentado de manera muy preocupante: en los últimos dos años ocurrieron 24, de los cuales 16 fueron por plaguicidas organofosforados (Martínez et al. 2009).

En su mayoría los estudios de entomotoxicología se enfocan a sustancias ilícitas como drogas (cocaína) y/o medicamentos (anfetaminas), pero en muy poca proporción se estudian los insecticidas, considerando que las intoxicaciones por estas sustancias son letales (Gunatilake y Goff 1989; Rumiza et al. 2008; Gosselin et al. 2011; Solís-Esquivel et al. 2016).

Los plaguicidas organofosforados, son sustancias que se relacionan de manera directa con asesinatos o suicidios, por tal motivo, en el presente estudio se optimizó un método analítico que permite la detección de malatión en larvas de mosca, como una herramienta indirecta para resolver posibles casos de homicidio.

\section{Materiales y métodos}

Lugar. La presente investigación fue realizada en el campo experimental de la Universidad Autónoma de Tamaulipas-
Unidad Aztlán (26³'45.49”N 98¹9'17.84”O) en la Cd. de Reynosa, Tamaulipas, México en el verano de 2017.

Sustrato. Goff y Lord (2010) señalan que el cerdo es la especie que más se aproxima a los patrones de descomposición cadavérica humana, por ello en la presente investigación se utilizó carne de ese animal como sustrato. Se utilizaron cuatro trozos de músculo de carne de cerdo Sus scrofa (Linnaeus) fresca sin congelar usando un diseño experimental con un control y un tratamiento (por triplicado). La dosis letal (DL) en humanos de malatión es de $60 \mathrm{~g} / 60 \mathrm{~kg}$, la cual fue convertida a dosis letal y se inyectó a $100 \mathrm{~g}$ de tejido de cerdo, de acuerdo a Liu et al. (2009); la carne fue expuesta al ambiente inmediatamente después de la aplicación. Se emplearon jaulas de metal, con la finalidad de evitar cualquier perturbación por agentes externos. La formulación del malatión utilizada fue (2[(dimetoxifosforotioil) sulfanil] butanodioatodedietilo) $\left(\right.$ Delta $^{\circledR}$ ) al 83,80\% y $16,20 \%$ de sus ingredientes inertes.

Matriz biológica. Al cuarto día de exposición se recolectaron larvas de tercer estadio las cuales previamente, se habían alimentado con carne tratada; posteriormente fueron sumergidas en agua caliente a $100{ }^{\circ} \mathrm{C}$ y, en seguida, lavadas con agua destilada. Se conservaron a $-20{ }^{\circ} \mathrm{C}$ hasta su procesamiento (Solís-Esquivel et al. 2016). Para el testigo (i.e., larvas alimentadas en carne sin insectida) se procesaron de manera similar. Otro grupo de larvas fue conservado en alcohol al $75 \%$, para propósitos de identificación.

Extracción. 1,5 g de material biológico (larvas de tercer estadio) se depositó dentro de un tubo eppendorf y una vez macerado se le agregó $1,2 \mathrm{~mL}$ de metanol $\left(\right.$ Tedia $\left.^{\circledR}\right)$-agua (70/30). Se agitó en vortex por espacio de $5 \mathrm{~min}$. Posteriormente, se centrifugó por $10 \mathrm{~min}$ a $10.000 \mathrm{rpm}$. Las muestras se filtraron en una membrana de $0,45 \mu$ (Merk-Millipore $\left.{ }^{\circledR}\right)$ y se transfirieron a viales de $2 \mathrm{ml}$ (Agilent Technologies ${ }^{\circledR}$ ).

Cromatografía. Se utilizó un equipo de cromatografía de líquidos de alta eficiencia HPLC (Agilent Serie 1100), la fase móvil fue $\left(\mathrm{CH}_{3} \mathrm{OH}\right): \mathrm{H}_{2} \mathrm{O}(70: 30)$ (Wang et al. 2012) se realizó la curva de calibración con estándar de malatión (Sigma ${ }^{\circledR}$ ) a $500,600,700,800,900$ y 1.000 partes por millón (ppm), a 30 ${ }^{\circ} \mathrm{C}$, flujo de $1 \mathrm{~mL} / \mathrm{min}$ y $220 \mathrm{~nm}$, columna RP C18 (Beckman Ultrasphere $^{\circledR}$ ).

Análisis de datos. Se utilizó análisis de varianza entre los tratamientos utilizando el programa JMP 13 (SAS Institute Inc. 2017).

\section{Resultados y discusión}

Sucesión de artrópodos. Un total de 369 individuos se recolectaron, todos del orden Diptera pertenecientes a tres familias y tres géneros (Tabla 1). Se observó que en el sustrato control la colonización fue más rápida ya que comenzó a las seis horas de haber colocado los sustratos, mientras que en la carne tratada la colonización fue después de 24 horas. Lo anterior coincide con lo reportado por Rumiza et al. (2008) quienes observaron que las larvas del grupo control se desarrollaron más rápidamente que las alimentadas con hígado que contenía malatión. Wolff et al. (2004) mencionan que las ovoposiciones se retrasan en sustratos tratados con paratión y Carvalho et al. (2000) especifican que el número 
Tabla 1. Diptera identificados en los sustratos de cerdo tratados con malatión.

\begin{tabular}{lcccc}
\hline \multicolumn{1}{c}{ Familia } & Género & Estado & Día & Muestra \\
\hline Sarcophagidae & Sarcophaga sp. & - & $0-8$ & Control \\
Calliphoridae & Chrysomya sp. & - & - & (Sin malatión) \\
Muscidae & Musca domestica L. & - & - & - \\
Sarcophagidae & Sarcophaga sp. & L3 & $4-8$ & Tratamiento 1 \\
Calliphoridae & Chrysomya sp. & L3 & 4-8 & Tratamiento 2 \\
\hline
\end{tabular}

de taxones disminuye cuando un cuerpo o un cadáver ha sido tratado con algún insecticida.

El proceso de estimación del intervalo post mortem podría ser erróneo si la presencia de malatión en las larvas no es considerado durante las primeras seis horas a 36 horas. Esto es apoyado para casos de intoxicaciones por malatión como fue reportado por Gunatilake y Goff (1989).

Por otra parte, los resultados obtenidos de los análisis en el HPLC, muestran que el tiempo de retención para la detección del malatión es a los 4,1 min (Fig. 1); aunque no hay un antecedente que estime este tiempo de retención por HPLC para este insecticida, lo más cercano es lo reportado por Wolff et al. (2004) quienes al analizar muestras de larvas de dípteros por este mismo método detectaron un tiempo de retención para el organofosforado paratión de 12,8 min. Otros análisis para malatión y paratión se han realizado en CG-DCE (cromatografía de gases-detección de captura de electrones), CG-SM (cromatografía de gases-acoplado a espectrometría de masas) y LC-DAD (cromatografía líquida-detector de diodos) (Gosselin et al. 2011).

Para la estimación de los resultados en las larvas, se realizó una curva de calibración usando un volumen de inyección de $20 \mu \mathrm{L}$ y tiempo de corrida $10 \mathrm{~min}$ (Fig. 2). La línea de regresión obtenida para la determinación de la curva de calibración fue $\mathrm{y}=9,124 \mathrm{x}+30,886$ y una $\mathrm{R}^{2}=0,9937$.

Los resultados del análisis por HPLC realizado a un grupo de larvas de tercer estadio para determinar el límite de detección (LDD) del insecticida concuerdan con lo obtenido por Mahat et al. (2012) quienes mencionan que la concentración de malatión incrementa gradualmente durante los estadios larvales encontrándose la máxima concentración en larvas de tercer estadio.

La concentración mínima de malatión fue calculada en 0,03010 ppm, en el caso del análisis por CG-EDC, Rumiza et al. (2008) encontraron $0,157,0,22$ y $0,045 \mu \mathrm{g} / \mathrm{mg}$ de malatión

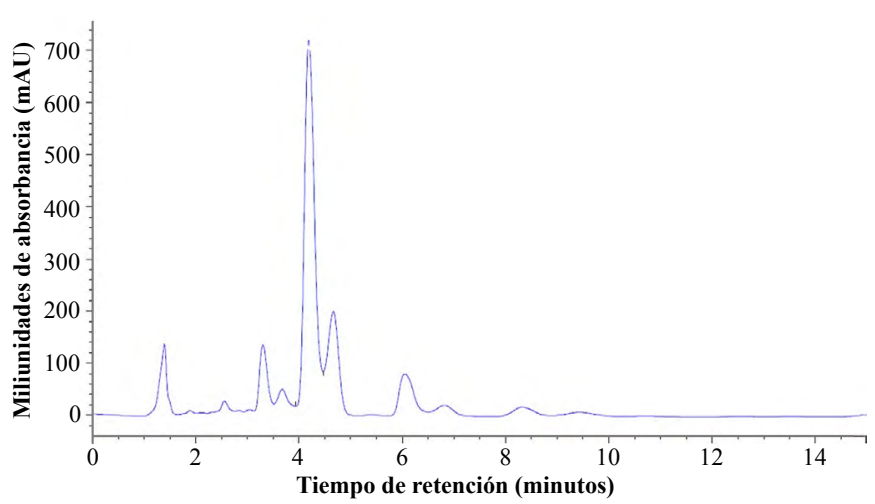

Figura 1. Cromatograma del estándar de malatión con un tiempo de retención de 4,1 usando una longitud de onda $220 \mathrm{~nm}$.

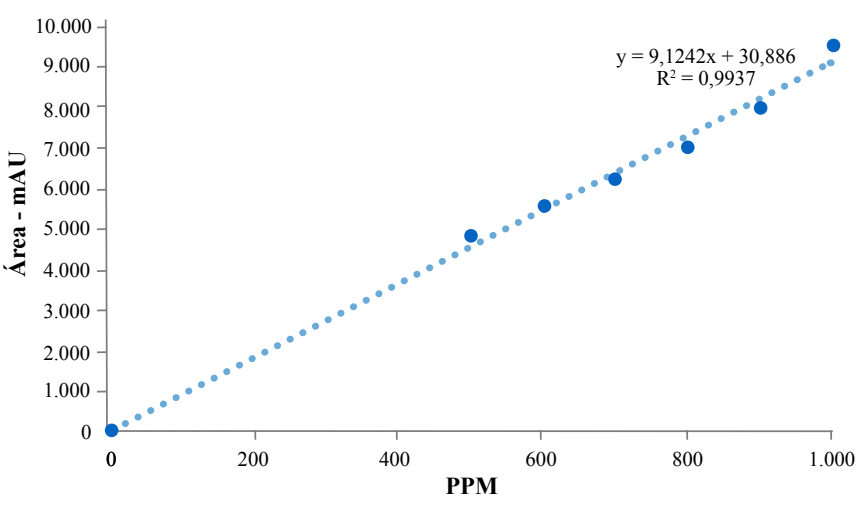

Figura 2. Curva de calibración desarrollada a partir de la información obtenida de las diferentes concentraciones de malatión que fueron analizadas por HPLC.

en larvas, pupas y adultos, respectivamente. Gunatilake y Goff (1989) reportaron en larvas de Chrysomia megacefala (Fabricius) y Chrysomia rufifacies (Macquart) una concentración de 2,050 $\mu \mathrm{g} / \mathrm{g}$. Mahat et al. (2012) reportaron 41,46 hasta $137,20 \mathrm{ng} / \mathrm{g}$ de concentración de malatión mientras que Liu et al. (2009) obtuvieron 8,53 hasta 28,90 ng/g de concentración de malatión en larvas de tercer estadio mientras que en pupas no fue detectado el compuesto. Lo anterior obedece a que de acuerdo con Silva et al. (2017) no existe una estandarización en este campo en cuanto a los métodos que se utilizan para llevar a cabo estas investigaciones, lo que ha llevado a la incapacidad de comparar los resultados entre los estudios.

Por otra parte, al realizar un análisis de la pérdida de malatión a través del tiempo, se tomó en cuenta la cantidad de insecticida con el cual las larvas estuvieron en contacto durante ocho días después de iniciado el tratamiento, siempre usando larvas de tercer estadio (96 horas de haber sido ovipositadas por la mosca) y que se alimentaron de la carne de cerdo tratada (Fig. 3). De estos datos se resalta que entre los días 4 y 7 se pierde el $50 \%$ de la capacidad de detección del insecticida en la carne tratada.

Los resultados muestran que no hay diferencia significativa entre los tratamientos, similar a lo reportado por Liu et al. (2009) quienes al analizar la mortalidad de acuerdo a las tasas de desarrollo, no detectaron diferencias significativas para las dosis letales de malatión de 1,0 y 1,5. Tampoco encontraron

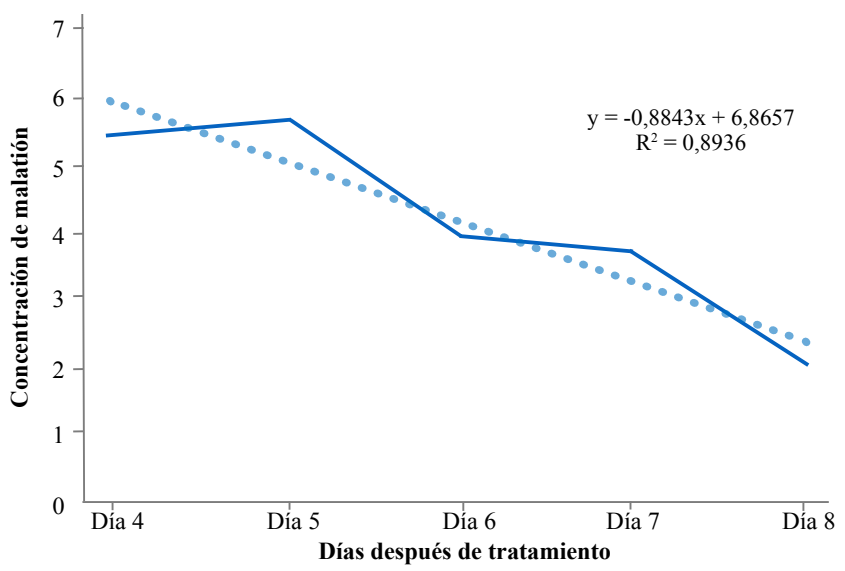

Figura 3. Línea de regresión de la velocidad de pérdida de malatión en larvas alimentadas con carne de cerdo tratada con insecticida. 
diferencia significativa entre el tratamiento control y los expuestos a la dosis letal 0,5 veces de malatión y plantean que la concentración de malatión en los tejidos afecta la cantidad residual en larvas y pupas que se alimentan de estos. Lo anterior puede ser causa de que el organofosforado no se distribuye de manera homogénea dentro del tejido y a que las larvas al alimentarse pueden comer de zonas con alta concentración, muy poca o incluso sin el insecticida como fue observado en este trabajo.

Por otro lado, los resultados indican que se pudo detectar eficientemente malatión. Estudios previos como el de Introna et al. (2001) señalan que una toxina puede ser detectada en las larvas cuando su tasa de absorción excede la de eliminación, lo cual también es confirmado por Anderson y VanLaerhoven (1996) y Goff et al. (1997) quienes enfatizan que las larvas de mosca se pueden alimentar de cualquier tejido que contenga compuestos químicos de cadáver.

Finalmente, Wolff et al. (2004) señalan que la cromatografía de líquidos de alta eficiencia (HPLC) es una técnica eficaz para detectar y cuantificar paratión en tejidos, así como en artrópodos presentes desde la fase inicial hasta los estados finales de descomposición incluyendo restos secos, tal como fue detectado en este trabajo.

\section{Conclusiones}

Los resultados muestran que es posible usar la técnica del HPLC para la detección de residuos de malatión aún después de varios días después de la exposición al insecticida. Por lo que es fundamental el uso de este instrumento en futuras investigaciones, con la finalidad de utilizarlo en casos judiciales como una herramienta en procesos en los que se sospeche la intoxicación por algún organofosforado.

\section{Agradecimientos}

Al Instituto Politécnico Nacional-Centro de Biotecnología Genómica y la Universidad Autónoma de Tamaulipas por las facilidades para el desarrollo del proyecto, a los miembros del Laboratorio de Biotecnología Experimental.

\section{Literatura citada}

ANDERSON, G.; VANLAERHOVEN, S. 1996. Initial studies on insect succession on carrion in southwestern British Columbia. Journal of Forensic Science 41 (4): 617-625. https://doi. org/10.1520/JFS13964J

ARNALDOS, M. I.; GARCÍA, M. D.; ROMERA, E.; PRESA, J. J.; LUNA, A. 2005. Estimation of post mortem interval in real cases based on experimentally obtained entomological evidence. Forensic Science International 149 (1): 57-65. https://doi. org/10.1016/j.forsciint.2004.04.087

CAMPOBASSO, C. P.; DI VELLA, G.; INTRONA, F. 2001. Factors affecting decomposition and Diptera colonization. Forensic Science International 120 (1-2): 18-27. https://doi.org/10.1016/ S0379-0738(01)00411-X

CARVALHO, L. M. L.; THYSSEN, P. J.; LINHARES, A. X.; PALHARES, F. A. B. 2000. A checklist of arthropods associated with pig carrion and human corpses in southeastern Brazil. Memórias do Instituto Oswaldo Cruz 95 (1): 135-138. https:// doi.org/10.1590/S0074-02762000000100023

GOEL, A.; AGGARWAL, P. 2007. Pesticide poisoning. National Medical Journal of India 20 (4): 182-191.

GOFF, M. L.; LORD, W. D. 2010. Insect as toxicological indicator and the impact of drugs and toxin on insect development. pp. 427-
436. En: Byrd, J. H.; Castner, J. L. (Eds.). Forensic entomology the utility of arthropods in legal investigations. Second edition. University of Georgia. CRC Press. Georgia. EE. UU. 682 p. https://doi.org/10.1201/NOE0849392153

GOFF, M. L.; MILLER, M. L; PAULSON, J. D.; LORD, W. D.; RICHARDS, E. OMORI, A. I. 1997. Effects of 3, 4 methylenedioxymethamphetamine in decomposing tissues on the development of Parasarcophaga ruficornis (Diptera: Sarcophagidae) and detection of the drug in postmortem blood, liver tissue, larvae and puparia. Journal of Forensic Science 42 (2): 276-280. https://doi.org/10.1520/JFS14110J

GOFF, M. L.; GARCIA, M. D.; ARNOLDOS, M. I.; LOZANO, R. E. 2004. Entomología cadavérica: fundamentos y aplicación. Referencia a la entomología española. pp. 253-262. En: Calabuig, J. A.; Villanueva, C. E. (Eds.). Medicina legal y toxicología. Sexta Edición. Barcelona, España. 1416 p.

GOSSELIN, M.; WILLE, S. M. R.; RAMÍREZ-FERNANDEZ, M.; DI FAZIO, V. SAMYN, N.; BOECK, G.; BOUREL, B. 2011. Entomotoxicology, experimental set-up and interpretation for forensic toxicologists. Forensic Science International 208 (1-3): 1-9. https://doi.org/10.1016/j.forsciint.2010.12.015

GUNATILAKE, K; GOFF, M. L. 1989. Detection of organophosphate poisoning in a putrefying body by analyzing arthropod larvae, Journal of Forensic Sciences 34 (3): 714-716. https://doi. org/10.1520/JFS12698J

INTRONA, F.; CAMPOBASSO, C. P.; GOFF, M. L. 2001. Entomotoxicology. Forensic Science International 120 (1-2): 4247. https://doi.org/10.1016/S0379-0738(01)00418-2

LIU, X.; SHI, Y.; WANG, H.; ZHANG, R. 2009. Determination of malathion levels and its effect on the development of Chrysomya megacephala (Fabricius) in South China. Forensic Science International 192 (1-3): 14-18. https://doi.org/10.1016/j. forsciint.2009.07.005

MAGAÑA, C. 2001. La entomología forense y su aplicación a la medicina legal: Data de la muerte. Boletín de la Sociedad Entomológica Aragonense (SEA) 28: 49-57.

MAGNI, P. A.; PACINI, T.; PAZZI, M.; VINCENTI, M.; DADOUR, I. R. 2014. Development of a GC-MS method for methamphetamine detection in Calliphora vomitoria $\mathrm{L}$. (Diptera: Calliphoridae). Forensic Science International 241: 96-101. https://doi.org/10.1016/j.forsciint.2014.05.004

MAHAT, N. A.; JAYAPRAKASH, P. T.; ZAFARINA, Z. 2012. Malathion extraction from of Chrysomya megacephala (Fabricius) (Diptera: Calliphoridae) for determining death due to malathion. Tropical Biomedicina 29 (1): 9-17.

MARTÍNEZ, R.; JARAMILLO, J.; ESCOTO, R.; RODRÍGUEZ, A.; POSADAS, R.; MEDINA, R. 2009. Preliminary comparative study of the necrophagous insects succession in Sus scrofa poisoned with methyl parathion, in three seasonal periods. Revista Mexicana de Ciencias Farmacéuticas 40 (3): 5-10.

OLIVA, A. 2001. Insects of forensic significance in Argentina. Forensic Science International 120 (1-2): 145-154. https://doi. org/10.1016/S0379-0738(01)00423-6

PIMENTEL, R. L.; GÁMIZ, W. A.; OLVERA, S. R.; SILVA, R. R. 2005. Panorama epidemiológico de las intoxicaciones en México. Medicina Interna de México 2: 123-132.

QUIROZ, M. H.; RODRÍGUEZ, C. A. 2015. Entomología forense, artrópodos y salud. Revista de Divulgación Científica (México) $2(2): 1-12$.

RUMIZA, A. R.; KHAIRUL, O.; MOHD, I.; RAJA, M. Z.; ROGAYAH, A. H. 2008. Determination of malathion levels and the effect of malathion on the growth of Chrysomya magachephala (Fabricius) in malathion-exposed rat carcass. Tropical Biomedicine 25 (3): 184-190.

SARMIENTO, V.; PADILLA, S. 2014. Entomological succession associated with decomposition processes in bodies of Oryctolagus cuniculus under field conditions. Sciéndo 17 (1): 134-140.

SAS Institute Inc. 2017. JMP (Versión 13). Cary NC, USA. 
SILVA, E. I. T.; WILHELMI, B.; VILLET, M. H. 2017. Forensic entomotoxicology revisited-towards professional standardization of study designs. International Journal of Legal Medicine 131 (2): 1399-1412. https://doi.org/10.1007/s00414-017-1603-9

SMITH, K. 1986. A manual of forensic entomology. The trustees of the British Museum (Natural History) and Cornell University Press. First Published. New York. 205 p.

SOLÍS-ESQUIVEL, E.; CABALLERO QUINTERO, A.; FLORES SUAREZ, A.; HERNÁNDEZ LUNA, C.; MERCADOHERNANDEZ, R.; RODRÍGUEZ-CASTRO, V. A.; QUIROZMARTINEZ, H. 2016. Detección de cocaína en larvas de dípteros necrófagos en Monterrey, Nuevo León, México. Southwestern Entomologist 41 (1): 99-104. https://doi. org/10.3958/059.041.0111

WANG, J.; XIA, Q.; ZHANG, A.; HU, X.; LIN, C. 2012. Determination of organophosphorus pesticide residues in vegetables by an enzyme inhibition method using $\alpha$ - naphthyl acetate esterase extracted from wheat flour. Journal of Zhejiang University Science B 13 (4): 267-273. https://doi.org/10.1631/ jzus.B11a0180

WOLFF, M.; URIBE, A.; ORTIZ, A.; DUQUE, P. 2001. A preliminary study of forensic entomology in Medellín, Colombia. Forensic Science International 120 (1-2): 53-59. https://doi.org/10.1016/ S0379-0738(01)00422-4

WOLFF, M.; BUILES, A.; ZAPATA, G.; MORALES, G.; BENECKE, M. 2004. Detection of parathion (O, O-diethyl
O-(4-nitrophenyl) phosphorothiolate) by HPLC in insects of forensic importance in Medellín, Colombia. Journal of Forensic Medicine and Toxicology 5 (1): 6-11.

\section{Origen y financiación}

El presente manuscrito se origina del trabajo de grado denominado "Análisis entomotoxicológicos para detección de malatión en larvas de dipteros de importancia forense". Este trabajo fue financiado parcialmente por la Universidad Autónoma de Tamaulipas y por el Instituto Politécnico Nacional y no existe conflicto de intereses en su publicación.

\section{Contribución de los autores}

Israel Estrada-Camacho: Desarrollo de protocolo, realización de los experimentos, toma de datos en campo e identificación de dipteros.

José Luis Hernández-Mendoza: Dirección del proyecto, participación activa en el manuscrito, interpretación y análisis de resultados.

Jorge Torres-Ortega: Análisis de las muestras entomológicas en el equipo HPLC.

Jesús Di Carlo Quiroz-Velázquez: Apoyo en la parte experimental, curvas de calibración y elaboración de gráficas.

Karla Villarreal-Sotelo: Participación en el análisis de la información y elaboración de manuscrito. 